\title{
Research on total least squares method based on error entropy criterion
}

\author{
Ben Wang ${ }^{a, *}$, Xiangyu Kong, Wei Huang and Zehao Cao \\ Xi'an Research Institute of High Technology, Xi'an, Shanxi 710025, China \\ a Corresponding author: 850939463@qq.com
}

Keywords: non-Gaussian; error entropy criterion (EEC); TLS; minimum total error entropy (MTEE).

\begin{abstract}
The total least square (TLS) estimation problem of random systems is widely found in many fields of engineering and science, such as signal processing, automatic control, system theory and so on. In the case of linear Gaussian case, a very mature TLS parameter estimation algorithm has been developed. In the non-Gaussian case, the existing research is not much and not deep, the current error entropy criterion (EEC) and the minimum error entropy( MEE) based on EEC method has been paid attention to, the traditional MEE only consider the output data contains the error situation, so it cannot get the optimal solution. In this paper, we consider the inclusion of noise in the input and output data, and deduce the total error entropy criterion (TEEC) and the corresponding TLS method named the minimum total error entropy (MTEE) method The In addition, the derivation method is simulated, and the simulation results show the correctness of the algorithm.
\end{abstract}

\section{Introduction}

The total least square (TLS) estimation problem of random systems is widely found in many fields of engineering and science, such as signal processing, automatic control, system theory and so on. Least squares method (least squares) is a classical method to solve this problem [1]. A basic assumption of the classical least squares method is that the noise is limited to the output data of the system. However, in the actual environment, there are many factors, such as sampling error, human error, and so on. In this case, the variable error (EIV) model can describe the input and output data more accurately. Due to the failure of the LS method, the biased estimation is applied to the EIV model.

The total least squares (TLS) method has been proposed as a solution to the problem of estimating the parameters of the [7] [5] EIV system in numerous solutions. The TLS method overcomes the shortcomings of the LS method, which takes into account the errors in the input and output data of the system, and takes the sum of squares of the fitting as the minimum "total" error. Currently in the classical EIV model, the TLS method has been shown to be superior to the LS method.

The existing TLS methods are based on the (total) minimum squared error, which depends on the two order statistics (general) error and the probability of reaching the optimal probability is Gauss. However, in real-world applications, non-Gauss noise is ubiquitous, for example, artificial noise in electronic devices, atmospheric noise and illumination spikes [6], [8]. Therefore, the probability distribution of error becomes non-Gauss. Therefore, in order to improve the performance of the estimation of the non-Gauss noise in practical applications, it is necessary to take into account the higher-order statistics of the error data.

This paper presents a general method of least squares in non-Gauss conditions. This method is based on the overall error entropy criterion, the reasonable consideration for the overall error is the case of Gauss, combined with the error entropy method in general, the total least squares method is applied to the non-Gauss case.

The rest of the paper is organized as follows: in section second, we present a concise statement of the current classical TLS method. In the third section, we introduce the total least squares method, which is applied to the non-Gauss condition. In the fourth section, the local stability of the proposed method is analyzed. The fifth section is the analysis of the experimental results. The sixth section is the conclusion. 


\section{Classical Total Least Squares Method}

The linear system is described by the equation:

$y_{i}=\boldsymbol{x}_{i} \boldsymbol{w}^{0}$

Where $\boldsymbol{w}^{0} \in \boldsymbol{R}^{D \times 1}$ is a parameter vector of the system, input $\boldsymbol{x}_{i} \in \boldsymbol{R}^{1 \times D}$, output $y_{i} \in \boldsymbol{R}, x, y$ are zero-mean Gaussian white noise.

At present, for the linear system only output with error, the input $x$ does not contain errors in the parameter estimation method has been a lot. Specific mathematical model

$\left\{\begin{array}{c}\breve{y}_{i}=y_{i}+v_{i} \\ \breve{x}_{i}=\boldsymbol{x}_{i}\end{array}\right.$

Where $v \in \boldsymbol{R}$ the zero-mean output of the covariance noise is is not related to the input.

Assuming that the above model is input with $N$ sets of data, many algorithms based on these data to estimate the system's parameter vector $\boldsymbol{w}$ are presented. The LS method based on the mean square error criterion is a more mature method to solve the problem of such parameters. The LS method mainly calculates the sum of the output errors,

$$
\begin{aligned}
& \min _{w} \sum_{i=1}^{i=N}\left(e_{i}^{\text {out }}\right)^{2} \\
& \text { Where } \\
& e_{i}^{\text {out }}=\breve{y}_{i}-\breve{\boldsymbol{x}}_{i} \boldsymbol{w}
\end{aligned}
$$

However, in the real environment, the noise is often non-Gaussian, LS method is not very practical. In this case, the general error entropy criterion (EEC) is used more [10] - [11], it can take into account all the error distribution rather than just the second order statistical error, the second order error statistics can not be sufficient The acquisition of non-Gaussian noise distribution. The minimum error entropy method (MEE) is an estimation method using the error entropy criterion (EEC). For the previous estimation problem, the error entropy method (MEE) is designed to minimize Renyi's entropy of the output error, as below,

$$
\min _{w} H_{\alpha}\left(e^{o u t}\right)=H_{\alpha}(\breve{y}-\breve{x} w)
$$

where $\alpha$ is the order of the Renyi's entropy and it is usually set to be 2.Although other values can also be used to compute the Renyi's entropy, taking $H_{\alpha=2}$ is better for computational complexity and feasibility.

The methods described above are based on the input data without error, the output data contains the assumption of error, but more of the input and output data are included in the error. Based on this situation, we use the variable error model (EIV), as follow,

$$
\left\{\begin{array}{l}
\breve{y}_{i}=y_{i}+v_{i} \\
\breve{\boldsymbol{x}}_{i}=\boldsymbol{x}_{i}+\boldsymbol{u}_{i}
\end{array}\right.
$$

Where $\boldsymbol{u}_{i} \in R^{1 \times D}$ zero-mean Gaussian is white noise and has stationary and ergodic. $\boldsymbol{x}_{i}, v_{i}$ are uncorrelated.

Unlike the LS method, the classical TLS method takes into account the input error and the output error in the linear system, that is, the following optimization problem,

$$
\begin{aligned}
& \min _{w,\left\{e_{i}^{\text {in }}\right\},\left\{e_{i}^{\text {out }}\right\}} \sum_{i=1}^{i=N} \varepsilon_{0}\left(e_{i}^{\text {out }}\right)^{2}+\left(e_{i}^{\text {in }} \varepsilon_{1}\right)^{2} \\
& \text { s.t. } \breve{y}_{i}-e_{i}^{\text {out }}=\left(\breve{\boldsymbol{x}}_{i}-e_{i}^{\text {in }}\right) \boldsymbol{w}, \quad \forall i \in\{1, \cdots, N\}
\end{aligned}
$$

Where $e_{i}^{i n} \in R^{1 \times D}$ is the input error and $\varepsilon=\left[\varepsilon_{0}, \varepsilon_{1}^{T}\right]=\left[\varepsilon_{0}, \varepsilon_{1}, \cdots, \varepsilon_{D}\right]$ is a weight vector of all positive terms. In [9], the author describes in detail the above optimization problem using singular value decomposition (SVD) method, This can prove the above optimization problems proven to be equivalent to the following minimization problem, 
$\min _{w} \sum_{i=1}^{i=N} \frac{\left|\breve{y}_{i}-\breve{x}_{i} \boldsymbol{w}\right|^{2}}{\boldsymbol{w}^{T} \Lambda_{1}^{-2} \boldsymbol{w}+\varepsilon_{0}^{-2}}$

Where $\Lambda_{1}=\operatorname{diag}\left(\varepsilon_{1}, \cdots, \varepsilon_{D}\right)$ is a nonsingular weighting matris.

By defining

$$
e^{t o t}=\frac{(\breve{y}-\breve{x} \boldsymbol{w})}{\sqrt{\boldsymbol{w}^{T} \Lambda_{1}^{-2} \boldsymbol{w}+\varepsilon_{0}^{-2}}}
$$

Equation (6) is simplified

$$
\min _{w} \sum_{i=1}^{i=N}\left(e_{i}^{\text {tot }}\right)^{2}
$$

Cost function of LS (3) and the cost function of TLS (11) in the same form. By comparing (3) and (11) it can be found that in the estimation, the LS method minimizes the sum of the squares of the output error, while the TLS method minimizes the sum of squares of the overall error.

It is easy to find that the LS method and the TLS method are based on the second order statistical error, so they cannot be effective for non-Gaussian noise. By comparing the LS method and the MEE method, we believe that in the non-Gaussian condition, the combination of TLS method and EEC criterion will be very effective. Under this idea, this paper deduces an overall minimum error entropy method (MTEE), which can deal with effectively the input and output contain the parameter estimation problem of non-Gaussian noise.

\section{Algorithm Formulation}

Based on the introduction of the previous section, in the EIV model non-Gaussian noise conditions, this paper estimates the system parameters by minimizing the quadratic Renyi's entropy of the total error $e^{\text {tot }}$, and we call the proposed algorithm the minimum total error entropy (MTEE) method. Specifically, the cost function is given by

$$
\begin{aligned}
J(\boldsymbol{w}) & =H_{\alpha=2}\left(e^{t o t}\right) \\
& =-\log \int p^{2}\left(e^{t o t}\right) d e^{t o t}
\end{aligned}
$$

Where ${ }^{p\left(e^{\text {tot }}\right)}$ is the probability distribution function (pdf) of $e^{\text {tot }}$.

Compared with LS method, TLS method and MEE method, MTEE method can be found on the basis of the first three methods. It can be regarded as the supplement and improvement of the first three methods.

Using the Parzen window method, the pdf $p\left(e^{\text {tot }}\right)$ can be estimated by $N$ sample of $e^{\text {tot }}$,

$$
\begin{array}{r}
\left\{e_{i}^{t o t}=\left(\breve{y}_{i}-\breve{x}_{i} w\right) /\left(\sqrt{\|w\|^{2}+\varepsilon_{0}^{-2}}\right), i=1, \cdots N\right\}, \\
\hat{p}\left(e^{t o t}\right)=\frac{1}{N} \sum_{i=1}^{n} G_{\sigma}\left(e^{t o t}-e_{i}^{t o t}\right)
\end{array}
$$

Where $G_{\sigma}(\cdot)$ is a Gaussian kernel function with a kernel size of $\sigma$. Substituting (13) into (12), you can get a simplified Renyi's entropy, as below,

$$
\hat{H}\left(e^{t o t}\right)=-\log \left(\frac{1}{N^{2}} \sum_{i=1}^{N} \sum_{j=1}^{N} G_{\sigma \sqrt{2}}\left(e_{j}^{t o t}-e_{i}^{t o t}\right)\right)
$$

Since the log function is monotonically increasing, minimizing the above estimator of quadratic Renyi's entropy is equivalent to maximizing the following term

$$
\hat{V}_{2}\left(e^{t o t}\right)=\frac{1}{N^{2}} \sum_{i=1}^{N} \sum_{j=1}^{N} G_{\sigma \sqrt{2}}\left(e_{j}^{\text {tot }}-e_{i}^{\text {tot }}\right)
$$

which is an estimator of the so-called quadratic information potential (QIP) [14]. Note that it can be viewed as the time average (or expectation) of $p\left(e^{t o t}\right)$, that is $\hat{V}_{2}\left(e^{t o t}\right)=\frac{1}{N} \sum_{i=1}^{N} p\left(e_{i}^{t o t}\right)$. 
Given the new cost function $\bar{J}(\boldsymbol{w})=\hat{V}_{2}\left(e^{\text {tot }}\right)$ ( $e^{\text {tot }}$ is a function of $\boldsymbol{w}$ ), we employ the steepest-ascent method to reach its maximum. Let us denote $\bar{\varepsilon}=\varepsilon_{0}^{-2}$ and take the derivative of (12), we have

$$
\nabla \bar{J}(\boldsymbol{w})=\frac{\partial \hat{V}_{2}\left(e^{t o t}\right)}{\partial \boldsymbol{w}}=\frac{\partial\left(\frac{1}{N^{2}} \sum_{i=1}^{N} \sum_{j=1}^{N} G_{\sigma}\left(e_{j}^{\text {tot }}-e_{i}^{t o t}\right)\right)}{\partial \boldsymbol{w}}=\frac{1}{\sigma^{2} N^{2}} \sum_{i=1}^{N} \sum_{j=1}^{N}\left[G_{\sigma}\left(e_{j}^{t o t}-e_{i}^{t o t}\right)\left(e_{i}^{\text {tot }}-e_{j}^{\text {tot }}\right) \times \frac{\partial\left(e_{j}^{\text {tot }}-e_{i}^{t o t}\right)}{\partial \boldsymbol{w}}\right]
$$

where

$$
\begin{aligned}
& \frac{\partial\left(e_{j}^{t o t}-e_{i}^{t o t}\right)}{\partial \boldsymbol{w}} \\
& =\frac{1}{\boldsymbol{w}^{T} \boldsymbol{w}+\bar{\varepsilon}}\left[-\left(\tilde{\boldsymbol{x}}_{j}^{T} \sqrt{\boldsymbol{w}^{T} \boldsymbol{w}+\bar{\varepsilon}}+\frac{\boldsymbol{w}}{\sqrt{\boldsymbol{w}^{T} \boldsymbol{w}+\bar{\varepsilon}}}\left(\tilde{y}_{j}-\tilde{\boldsymbol{x}}_{j} \boldsymbol{w}\right)\right)+\left(\tilde{\boldsymbol{x}}_{i}^{T} \sqrt{\boldsymbol{w}^{T} \boldsymbol{w}+\bar{\varepsilon}}+\frac{\boldsymbol{w}}{\sqrt{\boldsymbol{w}^{T} \boldsymbol{w}+\bar{\varepsilon}}}\left(\tilde{y}_{i}-\tilde{\boldsymbol{x}}_{i} \boldsymbol{w}\right)\right)\right] \\
& =\frac{1}{\sqrt{\boldsymbol{w}^{T} \boldsymbol{w}+\bar{\varepsilon}}}\left(\tilde{\boldsymbol{x}}_{i}^{T}-\tilde{\boldsymbol{x}}_{j}^{T}\right)+\frac{\boldsymbol{w}}{\boldsymbol{w}^{T} \boldsymbol{w}+\bar{\varepsilon}}\left(e_{i}^{\text {tot }}-e_{j}^{\text {tot }}\right)
\end{aligned}
$$

we denote $\left(e_{i}^{\text {tot }}-e_{j}^{\text {tot }}\right)$ as $\Delta e_{i j}^{\text {tot }}$, denote $\left(\tilde{\boldsymbol{x}}_{i}-\tilde{\boldsymbol{x}}_{j}\right)$ as $\Delta \tilde{\boldsymbol{x}}_{i j}$ and we suppress the superscript "tot" in the following derivation, in the case of no confusion. Using the simplified notations and combining (13) and (14) yields

$$
\frac{\partial \hat{V}_{2}\left(e^{t o t}\right)}{\partial \boldsymbol{w}}=\frac{1}{\sigma^{2} N^{2}} \sum_{i=1}^{N} \sum_{j=1}^{N} G_{\sigma}\left(\Delta e_{i j}\right)\left(\frac{\Delta e_{i j}^{2} \boldsymbol{w}}{\boldsymbol{w}^{T} \boldsymbol{w}+\bar{\varepsilon}}+\frac{\Delta e_{i j} \Delta \tilde{x}_{i j}^{T}}{\sqrt{\boldsymbol{w}^{T} \boldsymbol{w}+\bar{\varepsilon}}}\right)
$$

According to the steepest-ascent method, the iterative solution for optimizing $\bar{J}(w)$ is presented as

$$
\boldsymbol{w}_{\tau+1}=\boldsymbol{w}_{\tau}+\left.\mu \frac{\partial \tilde{V}_{2}(e)}{\partial \boldsymbol{w}}\right|_{\boldsymbol{w}_{\tau}}
$$

Where ${ }^{\tau}$ denotes the iteration step, and ${ }^{\mu}$ denotes the learning step-size.

The above results are obtained in the case of batch mode, where the $N$ data points are fixed during the estimation. In an online scenario, at time instant $i$, we can use the latest $L$ data points $\left\{\left(\tilde{\boldsymbol{x}}_{j}, \tilde{y}_{j}\right) \mid j=i-L+1, \cdots, i\right\}$ to calculate the latest $L$ samples of $e$, then estimate the QIP based on the $L$ samples. Furthermore, to reduce calculation cost, we approximate the estimate of QIP stochastically by dropping the time average in (12), leading to the expression [16]

$$
\hat{V}_{2}\left(e_{i}\right)=\frac{1}{L} \sum_{j=i-L+1}^{i} G_{\sigma \sqrt{2}}\left(e_{j}-e_{i}\right)
$$

By using the steepest-ascent method, we obtain the adaptive updating rule for the estimate of parameter vector:

$$
\boldsymbol{w}_{i+1}=\boldsymbol{w}_{i}+\left.\mu \frac{\partial \tilde{V}_{2}\left(e_{i}\right)}{\partial \boldsymbol{w}}\right|_{\boldsymbol{w}_{i}}
$$

Where

$$
\frac{\partial \hat{V}_{2}\left(e_{i}\right)}{\partial \boldsymbol{w}}=\frac{1}{\sigma^{2} L} \sum_{j=i-L+1}^{i} G_{\sigma}\left(\Delta e_{i j}\right)\left(\frac{\Delta e_{i j}^{2} \boldsymbol{w}}{\boldsymbol{w}^{T} \boldsymbol{w}+\bar{\varepsilon}}+\frac{\Delta e_{i j} \Delta \tilde{x}_{i j}^{T}}{\sqrt{\boldsymbol{w}^{T} \boldsymbol{w}+\bar{\varepsilon}}}\right)
$$

Next, we consider the computational complexity of the proposed MTEE algorithms. For the batch-mode MTEE, according to (18), in each iteration, the number of addition operation needed in calculating the gradient is proportional to $N^{2}$ and the number of multiply operation is proportional to the dimension of input signal, thus the computational cost is $O\left(D N^{2}\right)$. For the online-mode MTEE, in each iteration, the number of addition operation needed in calculating the gradient is proportional to the window length $L$ and the number of multiply operation is proportional to $D$, thus the computational cost is $O(D L)$.

Remark 1: In the development of the MTEE algorithm, the input/output measurement noise are merely assumed to be stationary and ergodic in time. Thus the MTEE can be applied in both independent identically distributed (i.i.d.) noise and correlated noise, so is the scaled TLS [3]. For correlated noise, we notice that the weighted TLS method can further improve the estimation 
performance by additionally considering the dependence (correlation) structure of measurement noise. Specifically, it introduces a weighting matrix, which is appropriately designed according to the covariance matrix of the noise, in its cost function [9]. However, such a trick cannot be simply employed in the MTEE, for the reason that the optimization problem of the weighted TLS cannot be efficiently solved by the SVD and from its cost function we can no longer educe a natural definition of total error as what we do from the scaled TLS. To the best of our knowledge, information theoretic learning specialized for correlated noise remains an unsolved and worth studying issue.

\section{Local Stability Analysis}

It is obviously that the local stability of the proposed algorithm is consistent with that of the TLS algorithm. In this section, we study the local stability of the proposed MTEE algorithm near the expected value of the parameter vector. In this paper, the stability of MTEE algorithm is analyzed, and the condition of local stability is obtained. Note that the performance surface of MTEE is non-quadratic and non-convex, so it is very difficult (if not impossible) to perform global stability and convergence analysis. Similarly, for the TLS algorithm, the theoretical analysis of global stability and convergence is also very difficult and rare. For the classical MEE method, there has been a lack of rigorous mathematical analysis over the past for a long period of time, and some theoretical analyzes have recently been conducted on consistency and convergence [13], [14], [16]. In general, the rigorous mathematical analysis of information theory learning methods is quite difficult and complex.

Assume that the estimate of the parameter vector is in the neighborhood of the local extreme point $w^{0}$ when the algorithm gets nearly converged (the convergence performance will be examined and studied by numerical simulations in the next section). By subtracting both sides of (16) from $w^{0}$, we get

$\overline{\boldsymbol{w}}_{\tau+1}=\overline{\boldsymbol{w}}_{\tau}-\mu \nabla \overline{\boldsymbol{J}}\left(\boldsymbol{w}_{\tau}\right)$

Where $\bar{w}_{\tau}$ is the error vector defined as

$\overline{\boldsymbol{w}}_{\tau}=\boldsymbol{w}^{0}-\boldsymbol{w}_{\tau}$

Since $\bar{J}(w)$ is twice continuously differentiable in a neighborhood of a line segment between points $w^{0}$ and $\boldsymbol{w}_{\tau}=\boldsymbol{w}^{0}-\overline{\boldsymbol{w}}_{\tau}$, by using the Theorem 1.2.1 of [16], we have

$$
\begin{aligned}
\nabla \bar{J}\left(\boldsymbol{w}_{\tau}\right) & =\nabla \bar{J}\left(\boldsymbol{w}^{0}\right)-\left[\int_{0}^{1} \boldsymbol{H}\left(\boldsymbol{w}^{0}-t \overline{\boldsymbol{w}}_{\tau}\right) d t\right] \overline{\boldsymbol{w}}_{\tau} \\
& \approx-\left[\int_{0}^{1} \boldsymbol{H}\left(\boldsymbol{w}^{0}-t \overline{\boldsymbol{w}}_{\tau}\right) d t\right] \overline{\boldsymbol{w}}_{\tau} \\
& \approx-\left[\int_{0}^{1} \boldsymbol{H}\left(\boldsymbol{w}^{0}\right) d t\right] \overline{\boldsymbol{w}}_{\tau} \\
& \approx-\boldsymbol{H}\left(\boldsymbol{w}^{0}\right) \overline{\boldsymbol{w}}_{\tau}
\end{aligned}
$$

Where $\boldsymbol{H}(\boldsymbol{w})$ is the Hessian matrix of $J(\boldsymbol{w})$. The first approximation follows from the fact $\nabla \bar{J}\left(\boldsymbol{w}^{0}\right) \approx 0$, which has been proved above. The second approximation follows from the assumption that $\boldsymbol{w}_{\tau}$ is in the neighborhood of $\boldsymbol{w}^{0}$ and thus $\overline{\boldsymbol{w}}_{\tau}$ is small. Substituting the result into (23), we obtain

$$
\overline{\boldsymbol{w}}_{\tau+1} \approx\left[\boldsymbol{I}+\mu \boldsymbol{H}\left(\boldsymbol{w}^{0}\right)\right] \overline{\boldsymbol{w}}_{\tau}
$$

Based on (26), to guarantee local stability, the magnitudes of all the eigenvalues of matrix $\left[\boldsymbol{I}+\mu \boldsymbol{H}\left(\boldsymbol{w}^{0}\right)\right]$ must be less than unity, leading to the following condition [3], [15]:

$\left|1+\mu \lambda_{d}\right|<1, d=1, \cdots, D$

Where $\left\{\lambda_{d}, d=1, \cdots, D\right\}$ are the eigenvalues of the Hessian matrix $\boldsymbol{H}\left(\boldsymbol{w}^{0}\right)$. At this point, the problem left is to calculate $\boldsymbol{H}\left(\boldsymbol{w}^{0}\right)$ and its eigenvalues.

To provide a clear and succinct calculation of $\boldsymbol{H}\left(\boldsymbol{w}^{0}\right)$, we first calculate the gradient vector (17) at the point $w^{0}$, as below 


$$
\boldsymbol{g}=\left.\frac{\partial\left(e_{j}^{t o t}-e_{i}^{t o t}\right)}{\partial \boldsymbol{w}}\right|_{w^{0}}=\frac{\Delta \varphi_{i j} \boldsymbol{w}^{0}}{a^{3 / 2}}+\frac{\Delta \tilde{\boldsymbol{x}}_{i j}^{T}}{a^{1 / 2}}
$$

And further calculate the gradient of (17) at the point $w^{0}$ as

$$
\begin{aligned}
\boldsymbol{Q} & =\left.\frac{\partial^{2}\left(e_{j}^{\text {tot }}-e_{i}^{\text {tot }}\right)}{\partial \boldsymbol{w}^{2}}\right|_{\boldsymbol{w}^{0}} \\
& =-\frac{\boldsymbol{w}^{0} \Delta \tilde{\boldsymbol{x}}_{i j}}{a^{3 / 2}}+\Delta \varphi_{i j} \frac{a \boldsymbol{I}-2 \boldsymbol{W}}{a^{5 / 2}}-\frac{\boldsymbol{w}^{0} \boldsymbol{g}^{T}}{a} \\
& =-\frac{2 \boldsymbol{w}^{0} \Delta \tilde{\boldsymbol{x}}_{i j}}{a^{3 / 2}}-\frac{3 \Delta \varphi_{i j} \boldsymbol{W}}{a^{5 / 2}}+\frac{\Delta \varphi_{i j} \boldsymbol{I}}{a^{3 / 2}}
\end{aligned}
$$

Where

$$
\boldsymbol{W}=\boldsymbol{w}^{0}\left(\boldsymbol{w}^{0}\right)^{T}
$$

Moreover, we define

$$
\begin{aligned}
\tilde{\boldsymbol{R}} & =E\left[\tilde{\boldsymbol{x}}_{i}^{T} \tilde{\boldsymbol{x}}_{i}\right] \\
& =E\left[\boldsymbol{x}_{i}^{T} \boldsymbol{x}_{i}\right]+E\left[\boldsymbol{u}_{i}^{T} \boldsymbol{u}_{i}\right] \\
& =\left(\sigma_{x}^{2}+\sigma_{i n}^{2}\right) \boldsymbol{I}
\end{aligned}
$$

So

$$
E\left[\Delta \tilde{\boldsymbol{x}}_{i j}^{T} \Delta \tilde{\boldsymbol{x}}_{i j}\right]=2 \tilde{\boldsymbol{R}}
$$

Based on the above results, the Hessian matrix $\boldsymbol{H}\left(\boldsymbol{w}^{0}\right)$ is calculated/approximated as (again, we use the approximation $G_{\sigma}\left(\Delta e_{i j}^{0}\right) \approx 1$ and use the expectation to replace the double time average in the following derivation)

\section{Simulation Results}

In this paper, we only validate the correctness of the MTEE algorithm, that is, whether the direction cosine of the algorithm can converge to 1 . Only the direction cosine of the parameter estimation algorithm can converge to 1 , which can explain the correctness of the proposed algorithm. On the same time, only the direction cosine of the algorithm converges to 1 to carry on the other performance indexes of the algorithm on this basis Proof and analysis. The MTEE algorithm proposed in this paper can only prove the correctness of the algorithm and deduce the local stability, and the comparison of the effect of the algorithm cannot be completed.

In the simulations of this subsection, we set the total number of data samples as 300 and the maximum number of iteration as 100. Besides, for the MTEE and MEE, we let their learning step-sizes slowly decrease from 0.05 to 0.005 during the iterations, respectively. The initial guess of the system vector for all the methods is set as a 51 zero vector. If not otherwise specified, the kernel size is set as 0.5 , which is slightly smaller than the input signal intensity. All the results shown in this part are calculated over 500 independent runs

It can be seen from the figure that the direction cosine of MTEE can converge to 1 very quickly, and the validity of the algorithm is explained. At the same time, any one of the data points of the algorithm is above 0.8 , which indicates that the algorithm is very stable Excellent, the whole process is very smooth curve, but also reflects the robust performance of the algorithm. 


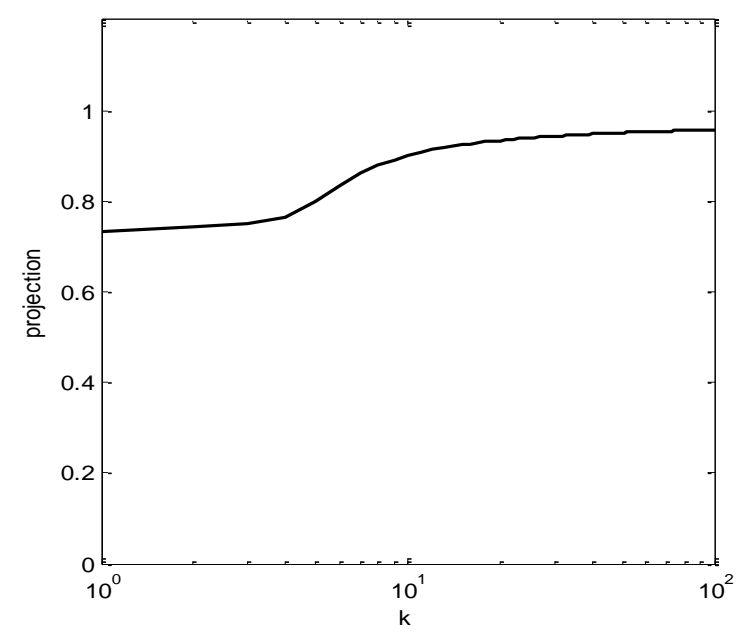

Figure 1. To format a figure caption use the Microsoft Word template style.

\section{Conclusion}

In this paper, a TEEC-based stochastic system TLS parameter estimation algorithm is proposed, called MTEE method. It is a supplement to existing LS methods, TLS methods, and MEE methods. From the geometric point of view, the main difference between MTEE and MEE is that they use two different distance measures in the cost function, which is the same as the difference between TLS and LS. However, MTEE and MEE consider the entropy of the distance, while TLS and LS consider the second-order statistics of the distance. On the basis of the TLS method, the MTEE method also considers the non-Gaussian noise, and the MTEE further considers the existence of the input noise compared with the MEE method. Therefore, MTEE will get better estimates than TLS and MEE for EIV systems under non-Gaussian noise. We have developed MTEE methods for batch mode and online mode. In the online model MTEE, we randomly approximate the gradient, so that the algorithm is computationally efficient. It has been shown that the algorithm is locally stable around the specific values of the parameters under certain conditions. Based on the theoretical derivation, the MTEE estimation is better than the other three methods in the non - Gaussian case, and the correctness of the MTEE algorithm is verified in the further experimental simulation. In the next step of the study, will be in-depth study MTEE algorithm, the MTEE algorithm performance and algorithm features carefully analyzed, and combined into practical applications. .

\section{Acknowledgments}

Thanks the mentor of the National Natural Fund surface project: Research on Key Technology of Data-driven Random System Signal Feature Extraction and Performance Degradation Modeling (61374120); Data-driven process data modeling and application of missile control fault diagnosis (61673387)

\section{References}

[1]. X. Kong, C. Han, and R. Wei, "Modified gradient algorithm for total least square filtering," Neurocomputing, vol. 70, pp. 568-576, Dec. 2006.

[2]. R. Arablouei, S. Werner, and K. Dogancay, "Analysis of the gradient descent total least-squares adaptive filtering algorithm," IEEE Trans. Signal Process. vol. 62, no. 5, pp. 1256-1264, Mar. 2014.

[3]. S. Van Huffel and P. Lemmerling, Total Least Squares Techniques and Errors-in-Variables Modeling: Analysis, Algorithms and Applications. Dordrecht, the Netherlands: Kluwer Academic, 2002. 
[4]. T. Soerstrom, "Errors-in-variables methods in system identification," Automatica, vol. 43, pp. 939-58, 2007.

[5]. R. Arablouei, K. Dogancay, and T. Adali, "Unbiased recursive least-squares estimation utilizing dichotomous coordinate-descent iterations,"IEEE Trans. Signal Process., vol. 62, no. 11, pp. 2973-2983, Jun. 2014.

[6]. G. H. Golub and C. F. Van Loan, "An analysis of the total least squares problem," SIAM J. Numerical Anal., vol. 17, no. 6, pp. 883-893, Dec. 1980.

[7]. B. Chen, Y. Zhu, and J. Hu, "Mean-square convergence analysis of ADALINE training with minimum error entropy criterion," IEEE Trans. Neural Netw., vol. 21, no. 7, pp. 1168-1179, Jul. 2010.

[8]. J. C. Principe, Information Theoretic Learning: Renyi's Entropy and Kernel Perspectives. Berlin, Germany: Springer-Verlag, 2010.

[9]. D. Erdogmus and J. C. Principe, "Generalized information potential criterion for adaptive system training," IEEE Trans. Neural Netw., vol. 13, no. 5, pp. 1035-1044, May 2002.

[10]. D. Erdogmus and J. C. Principe, "Comparison of entropy and mean square error criteria in adaptive system training using higher order statistics,"'in Proc. Independent Components Analysis (ICA), Helsinki, Finland, 2000.

[11]. D. Erdogmus and J. C. Principe, "Convergence properties and data efficiency of the minimum error entropy criterion in ADALINE training," IEEE Trans. Signal Process., vol. 51, no. 7, pp. 1966-1978, Jul. 2003.

[12]. B. E. Dunne and G. A. Williamson, "Stable simplified gradient algorithms for total least squares filtering," in Proc. Conf. Record 34th Asilomar Conf. Signals, Syst., Comput., 2000, vol. 2, pp. 1762-1766.

[13]. B. E. Dunne and G. A. Williamson, "Analysis of gradient algorithms for TLS-based adaptive IIR filters,” IEEE Trans. Signal Process., vol. 52, no. 12, pp. 3345-3356, Dec. 2004.

[14]. X.-L. Li and T. Adali, "Flexible adaptive filtering by minimization of error entropy bound and its application to system identification," presented at the ICASSP, Dallas, TX, USA, 2010.

[15]. H.-L. Li, X.-L. Li, M. Anderson, and T. Adali, "A class of adaptive algorithms based on entropy estimation achieving CRLB for linear non-Gaussian filtering," IEEE Trans. Signal Process., vol. 60, no. 4, pp. 2049-2055, Apr. 2012.

[16]. C. Li, P. Shen, Y. Liu, and Z. Zhang, "Diffusion information theoretic learning for distributed estimation over network," IEEE Trans. Signal Process. vol. 61, no. 16, pp. 4011-4024, Aug. 2013.

[17]. T. Hu, J. Fan, Q. Wu, and D. X. Zhou, "Learning theory approach to minimum error entropy criterion,” J. Mach. Learn. Res., vol. 14, pp. 377-397, 2013.

[18]. T. Hu, J. Fan, Q. Wu, and D. X. Zhou, "Regularization schemes for minimum error entropy principle," Anal. Appl., vol. 13, pp. 437-455, 2015.

[19]. R. Jenssen, D. Erdogmus, K. E. Hild, J. C. Principe, and T. Eltoft, "Information cut for clustering using a gradient descent approach," Pattern Recognit., vol. 40, no. 3, pp. 796-806, 2007.

[20]. M. Mojiri and A. R. Bakhshai, "An adaptive notch filter for frequency estimation of a periodic signal,” IEEE Trans. Autom. Control, vol. 49, no. 2, pp. 314-318, Feb. 2004.

[21]. L. Tan and J. Jiang, "Novel adaptive IIR filter for frequency estimation and tracking," IEEE Signal Process. Mag., vol. 26, no. 6, pp. 186-189, Nov. 2009.

[22]. C. Li and H. Wang, "Distributed frequency estimation over sensor network," IEEE Sensors J., vol. 15, no. 7, pp. 3973-3983, Jul. 2015. 